Religiöse Strategien in Pandemien und theologische Deutungen von Seuchen in Zeitgeschichte und Gegenwart : Internationale Forschungsperspektiven und Fragestellungen

Kunter, Katharina

2021

Kunter , K 2021 , ' Religiöse Strategien in Pandemien und theologische Deutungen von Seuchen in Zeitgeschichte und Gegenwart : Internationale Forschungsperspektiven und Fragestellungen ' , Theologische Rundschau. , vol. 86 , no. 3/4 , pp. 331-337 . https://doi.org/10.1628/thr-2021-0017

http://hdl.handle.net/10138/340095

https://doi.org/10.1628/thr-2021-0017

unspecified

acceptedVersion

Downloaded from Helda, University of Helsinki institutional repository.

This is an electronic reprint of the original article.

This reprint may differ from the original in pagination and typographic detail.

Please cite the original version. 


\title{
Religiöse Strategien in Pandemien und theologische Deutungen von Seuchen in Zeitgeschichte und Gegenwart:
}

\section{Internationale Forschungsperspektiven und Fragestellungen}

\author{
Katharina Kunter, Theologische Fakultät der Universität Helsinki
}

Religion ist in der nun bereits eineinhalb Jahre andauernden Covid-19-Pandemie weltweit in zwei gegenläufigen Erscheinungsformen sichtbar geworden: Auf der einen Seite als Superspreader innerhalb religiöser - oft religiös-fundamentalistischer - Gemeinschaften und damit verbunden als ein Gegner weltlich-vernünftiger staatlicher und öffentlich unterstützter Pandemie-Maßnahmen oder Präventionen, wie etwa den Abstandsregelungen und Kontakteinschränkungen bei religiösen Zusammenkünften. Darüber hinaus sind Mitglieder dieser Gemeinschaften häufig in aktuellen transnationalen "Anti-ImpfBewegungen" aktiv und sichtbar. Auf der anderen Seite zeigte sich Religion in der CoronaKrise zugleich als ein globaler ziviler Akteur. Vor allem im Globalen Süden sind Religion und Religionsgemeinschaften ein integraler Bestandteil der Identität der Menschen und beeinflussen somit ihre Überzeugungen und Praktiken - wie auch die Maßnahmen, die sie zur Krisenprävention anwenden oder ablehnen. So vermochte es Religion, Individuen, Familien und Gemeinschaften in einer Situation größter existenzieller und sozialer Not mit Vertrauen, Zuversicht und konkreter Fürsorge und Hilfsangeboten zu stabilisieren. Nicht zuletzt bedeutet Religion Alltagspraxis in zahlreichen unterprivilegierten und marginalisierten gesellschaftlichen Gruppen, zu denen staatliche Informationspolitik oft keinen Zugang findet. Glaubensführer in zahlreichen Ländern trugen mit dazu bei, genaue und zuverlässige Informationen zum Covid-19-Virus zu verbreiten und wirkten damit Gerüchten, Falschinformationen oder Verschwörungstheorien entgegen. Diese beiden Seiten gehören zum Erscheinungsbild gegenwärtiger Religion in der Pandemie. 
In einer Welt, in der sich über 80\% der Weltbevölkerung (mit steigender Tendenz) mit einer religiösen Gruppe identifizieren ${ }^{1}$, besitzt das Zusammenwirken von Religion und Pandemie also eine globale Dimension, die weder von Politik, Wissenschaft und Religionsführern und deren Organisationen ausgeblendet werden kann.

Angesichts dieses Befundes ist es freilich auffällig, wie wenig die Rolle von Religion bei Pandemien und Krisenprävention bisher in Wissenschaft, Öffentlichkeit und auch unter den Religionsführern und Kirchenvertretern analysiert und diskutiert wurde. ${ }^{2}$ Drei Aspekte scheinen dafür maßgeblich zu sein: 1) Die wissenschaftliche, öffentliche und kirchliche Diskussion wurde wissenschaftlich durch virologische und epidemiologische Kenntnisse und Interpretationen ${ }^{3}$ bestimmt und politisch gerahmt durch ökonomische Überlegungen; 2) bis zum Ausbruch des Coronavirus gab es nur wenige empirische Studien, die wissenschaftlich kompatible, differenzierte Aussagen über die Rolle von Religion bei Seuchen und Pandemien, auch in einer historischen Perspektive, ermöglichten; 3) sieht man von Deutschland als einem Sonderfall ab, gibt es in den modernen westlichen Gesellschaften eine Hemmschwelle, Religion in Politik und Öffentlichkeit zu diskutieren, weil Religion oft als privat, irrational, emotional oder gar antimodern im säkularen Sinne wahrgenommen wird. Die Grenzen zur Verletzung des Rechts auf Religionsfreiheit sind fließend. Gleichzeitig kollidiert das Recht auf Religionsfreiheit in Pandemie-Situationen mit dem Recht auf Gesundheit, wie es 1946 von der WHO und 1966 vom UN-Sozialpakt formuliert wurde. ${ }^{4}$

Die hier kurz zusammengefassten Beobachtungen bilden den Ausgangspunkt für einen interdisziplinären deutsch-niederländisch-finnischen Forschungsverbund, der in Kooperation mit der Arbeitsgruppe „Religion and Pandemics“ der European Academy of Religion (EuARe) das Zusammenwirken von Religion und Pandemie an ausgewählten historischen und

\footnotetext{
${ }^{1}$ PEW Research Center, 2012: The Global Religious Landscape. https://www.pewforum.org/2012/12/18/globalreligious-landscape-exec/

${ }^{2}$ Siehe auch Befunde bei Wischmeyer, Johannes, 2020: Umgang der Religionen mit der Corona-Krise:

Evangelische Kirchen und ihr Umfeld: https://www.uni-muenster.de/Religion-und-

Politik/aktuelles/schwerpunkte/epidemien/04 thema verschwoerung.html und Universität Münster, 2020:

Religiöse Deutungen von Epidemien, https://www.uni-muenster.de/Religion-und Politik/aktuelles/schwerpunkte/epidemien/i Religioese Deutungen von Epidemien.htm

${ }^{3}$ Vgl. Wildman, Weseley J., Bulbulia, Joseph, Sosis, Richard; Schjoedt, Uffe, 2020: Religion and the COVID-19 pandemie. Religion, Brain and Behavior 2, 115-117.

${ }^{4}$ Vgl. Zuniga, José, Marks, Stephen P., Gostin, Lawrence O. (Hg.), 2013: Advancing the Human Right to Health. Oxford: Oxford University Press sowie Tobin, John, 2012: The Right to Health in International Law. Oxford: Oxford University Press 2012, 30-34.
} 
aktuellen Fallbeispielen eingehender untersuchen möchte. Er orientiert sich an dem Konzept der "Soft Power" aus der Theorie der Internationalen Beziehungen, das bereits in den 1980er Jahren von dem amerikanischen Politikwissenschaftler Joseph Nye eingeführt wurde. ${ }^{5}$ Zwar entwickelte Nye sein Konzept ursprünglich für den Bereich der Außenpolitik; in diesem Kontext erfuhr es auch unterschiedliche Kritik. Doch gerade der Einwand, Nyes Konzept sei zu vage und allgemein ${ }^{6}$, trägt der Pluralität religiöser Ausdrucksformen, Haltungen und Gemeinschaften angemessener Rechnung als eine vereinheitlichende Kategorisierung und macht es zu einem interdisziplinär anschlussfähigen Analyserahmen. Religion wird als eine kulturelle Agentur verstanden, die Mentalitäten, Werte und Handlungen ohne politischen Druck beeinflussen kann. ${ }^{7}$

Als übergreifende Leitperspektive untersuchen die Fallbeispiele, welche theologischen Deutungsmuster Religionen zu Seuchen entwickeln und inwieweit Religion als ein spezifischer nichtstaatlicher sozialer Akteur innerhalb globaler Pandemien verstanden werden kann. ${ }^{8}$ Der Forschungsverbund fragt weiterhin, welche gesellschaftlichen Wirkungen sich aus diesen religiösen Deutungen und Verhaltensweisen in verschiedenen Epochen und Kontexten nachweisen lassen und ob - und inwiefern - sich Religion tatsächlich als eine „Soft Power" in regionalen Epidemien, Seuchen und globalen Pandemien identifizieren lässt. Die Fallbeispiele beziehen sich forschungspraktisch bedingt zunächst überwiegend auf das Christentum, streben aber für die Zukunft eine breitere multireligiöse Perspektive und wissenschaftliche Vernetzung an. Im Einzelnen handelt es sich um die folgenden vier ausgewählten Forschungsthemen:

1) Leon van der Broeke (VU Amsterdam / Theologische Universiteit Kampen) untersucht die "Groninger-ziekte" (Groninger Krankheit), eine Art Malaria, die in den 1820er Jahren nicht nur in der Provinz Groningen im Nordosten der Niederlande, sondern auch in anderen

\footnotetext{
${ }^{5}$ Nye, Joseph, 1991: Bound to lead: The Changing Nature of American Power, New York: Basic Books sowie Ders., 2005: Soft Power: The Means to Success in World Politics. New York: Public Affairs..

${ }^{6}$ Hall, Todd, 2010: An Unclear Attraction: A Critical Examination of Soft Power as an Analytical Category, The Chinese Journal of International Politics, Bd. 3,189-211.

${ }^{7}$ Kunter, Katharina, 2015: Die Kirchen und die neue globale Hilfsgesellschaft: Die Gründung des Steering Commitee for Humanitarian Response (SCHR) 1972. Damberg, Wilhelm, Jähnichen, Traugott (Hg.), Neue Soziale Bewegungen als Herausforderung sozialkirchlichen Handelns, 245-261:248.

${ }^{8}$ Siehe auch Ferris, Elizabeth, 2005: Faith-based and secular humanitarian organizations. International Review of the Red Cross, Bdl. 87, 311-325.
} 
Provinzen und in nördlichen Teilen Deutschlands wütete. Durch sie wurden 10 \% der Einwohner der Provinz Groningen getötet. Vor dem Hintergrund der Covid-19 Pandemie stellen sich hier neue Fragen an das Quellenmaterial: Wie gingen die religiösen Führer, die Generalversammlungen der örtlichen Glaubensgemeinschaften und die Anhänger der Glaubensgemeinschaften mit dieser Epidemie um? Wie beschrieben und deuteten sie das Leid und die zahlreichen Toten, wie betteten sie ihren eigenen Tod in die Epidemie ein? Diese Fragen sollen aus historischer, religionsgeschichtlicher und kirchenpolitischer Sicht beantwortet werden.

2) Die zweite historische Fallstudie ist bei Katharina Kunter (Theologische Fakultät Helsinki) angesiedelt und richtet sich auf Finnland und Deutschland in den 1950er und 1960er Jahren. Sie untersucht, wie die christlichen Kirchen mit zwei der schlimmsten Pandemien dieser Zeit umgingen: der Asiatischen Grippe, die in Finnland 1.800 Menschen und in Deutschland 30.000 Menschen tötete und der Hongkong-Grippe, die in Finnland 1.000 Menschen und in Deutschland ebenfalls 30.000 Menschen tötete. Wie kommunizierten Gläubige und Kirchen die Asiatische Grippe und die Hongkong-Grippe innerhalb ihrer eigenen Gemeinschaft? Mit welchen religiösen und theologischen Motiven wurde die Grippe interpretiert, z. B. als Strafe Gottes? Wie sahen die religiösen Akteure in den 1950er und 1960er Jahren die Gesundheitsprävention, wie wurde sie in ihr eigenes religiöses Weltbild eingeordnet? Gab es Unterschiede zwischen lutherischen, reformierten, katholischen oder orthodoxen Christen, in den Interpretationen zwischen den Geschlechtern? Mit welchen Erfahrungen waren die Pfarrer und Krankenhausseelsorger konfrontiert, wie ging man mit den Toten um, wie waren etwa Beerdigungen und andere Kasualien organisiert? Ein besonderes Augenmerk gilt weiterhin den Impfungen als einem wichtigen Instrument der Pandemieprävention. Gab es in den 1950er und 1960er Jahren religiös oder theologisch argumentierende Impfgegner? Und inwiefern wirkten die noch existenten Volkskirchen hier prägend? Die Fallstudie ist bei Katharina Kunter (Theologische Fakultät Helsinki) angesiedelt. Historisch ist dabei zu bedenken, dass die finnische und deutsche Gesellschaft der 1950er und 1960er Jahre religiös homogener war. Zugleich setzten sich in dieser Zeit in Westeuropa jedoch umfassende Säkularisierungsprozesse in der Gesellschaft durch, die zu religiöser Modernisierung in den religiösen Institutionen, also den Kirchen, führten. Anhand der Quellen soll daher auch gefragt werden, inwiefern sich Religion aus diesem 
Säkularisierungsdruck heraus auf Seuchen- und Krisenprävention trotz des Paradoxes der Moderne einließ. ${ }^{9}$ Hier verdient die Tatsache, dass die finnische Bevölkerung die höchsten Zustimmungsraten zu staatlich organisierten Massenimpfungen in Europa besitzt und die höchsten Raten von Menschen, die diese als mit ihrer Religion vereinbar ansehen, aufweist, besondere Aufmerksamkeit. ${ }^{10}$ Inwiefern ist dies auch ein Ergebnis einer besonderen religiösen (vielleicht spezifisch lutherischen?) Kommunikation über Krisenvorsorge? Die Untersuchung dieses Aspektes ist auch für den gegenwärtigen interreligiösen Dialog von Bedeutung: Denn fast alle Religionen kennen den Gedanken, dass sich die Gläubigen keine Sorgen und Gedanken um die Zukunft machen brauchen, da Gott für sie sorgt. Im Christentum ist dieser Gedanken z.B. im Neuen Testament bei Matthäus 6, 34 verankert. Will man heute auch stärker fundamentalistische oder streng an den Heiligen Schriften orientierte religiöse Akteure für die Seuchen- und Krisenprävention gewinnen, ist die Einsicht in die historischen Wandlungsfähigkeit von Normen und Werten zentral.

3) Die dritte historische Fallstudie analysiert die transnationale Interaktion religiöser Akteure in Finnland, Deutschland, den Niederlanden und der Ökumene einerseits und Westafrika andererseits im Kampf gegen die Ebola-Virus-Epidemie von 2014 bis 2016. Im Vergleich zur relativ homogenen religiösen Landschaft der 1950er und 1960er Jahre in Deutschland, Finnland und in den Niederlanden zeigte sich nun ein sehr viel breiter gefächertes religiöses Feld, denn neben einer Vielzahl von christlichen Kirchen, Gemeinschaften und Organisationen waren jetzt auch sowie muslimische und andere religiöse Akteure und religiöse humanitäre oder ökumenische Organisationen und NGOs wie ACT Alliance oder dem Ökumenischen Rat der Kirchen in den betroffenen Gebieten Afrikas aktiv. Welche Informationskanäle nutzen sie, welche Strategien und Maßnahmen leiteten sie ein, um die Epidemie einzudämmen? Und wie wurde die Ebola-Virus-Epidemie in Nordeuropa und in Westafrika religiös interpretiert; spielten auch Genderaspekte eine Rolle? Welche religiösen Einstellungen und Mentalitäten veränderten sich im Vergleich zu den 1950er und 1960er Jahren und dem 19. Jahrhundert (hier sind auch Bezüge zu Forschungen zur Spanischen Grippe denkbar)?

\footnotetext{
${ }^{9}$ Vgl. Hannig, Nicolai, 2019: Kalkulierte Gefahren. Naturkatastrophen und Prävention seit 1800, Göttingen: Wallstein.

${ }^{10}$ Siehe Euopeandata, 2019: https://www.europeandatajournalism.eu/eng/News/Data-news/Europe-svaccine-issue-the-fault-of-anti-vaxers-or-religion
} 
4) Der letzte Forschungsbereich bezieht sich auf aktuelle Fragen zu „Religion, Pandemie und Global Governance“ und erhält mit zwei digitalen Panels auf der European Academy of Religion im August 2021 einen breiteren diskursiven Rahmen. ${ }^{11}$ Weil alle religiösen Akteure weltweit von der Schwere und der globalen Dimension der Corona-Krise im März 2020 überrascht und in vielfältiger Weise an der humanitären Hilfe und der Seelsorge vor Ort beteiligt waren, vermittelte sich in Medien und Öffentlichkeit oft der Eindruck, dass das spirituelle Potenzial der christlichen Kirchen in Zeiten von Pandemien unsichtbar geworden sei. Das erste Panel greift diese Kritik an der Unsichtbarkeit der Glaubensgemeinschaften während der COVID19-Pandemie auf und erörtert aus internationaler evangelischer und katholischer Sicht die gesellschaftspolitische, sozialethische und moralische Verantwortung sowie das seelsorgerliche Engagement der Kirchen in der Covid-19-Krise. ${ }^{12}$ Aufbauend auf den theologischen Ansätzen zur sozialethischen und gesellschaftlichen Verantwortung von Kirchen und Religionen, untersucht dann das zweite Panel die globalen und humanitären Strategien, mit denen die verschiedenen religiösen Akteure auf vergangene Pandemien und die aktuelle Corona-Pandemie reagiert haben. Zum einen werden verschiedene christlichkonfessionelle Initiativen miteinander verglichen. Zum anderen wird analysiert, wie im 21. Jahrhundert religiöse Entwicklungsarbeit und globale Krisenprävention zusammenwirkten. Ein weiterer Schwerpunkt der Diskussion ist die Verflechtung und Verbindung der katholischen Kirche und globaler protestantischer humanitärer Organisationen mit den Organisationen der Vereinten Nationen (UN). Denn es ist auffallend, dass sich in jüngster Zeit zentrale Einrichtungen und Organe der UN, die sich ja als eine säkulare humanitäre Organisation versteht, besonders um religiöse Führungen und Organisationen als Partner in der Pandemie bemüht haben. So wies etwa Michelle Bachelet, die Hohe Kommissarin für Menschenrechte der $\mathrm{UN}^{13}$, verschiedene Vertreter der Weltgesundheitsorganisation

\footnotetext{
${ }^{11}$ Siehe https://www.europeanacademyofreligion.org/euare2021

${ }^{12}$ Vgl. Bachelet, Michelle, 2020: Global Pledge for Action by Religious Actors and Faith-Based Organizations to Address the COVID-19 Pandemic in Collaboration with the United Nations. https://www.ohchr.org/EN/NewsEvents/Pages/DisplayNews.aspx?NewsID=25909\&LangID=E https://www.europeanacademyofreligion.org/euare2021

${ }^{13}$ Bachelet, Michelle, 2020: Global Pledge for Action by Religious Actors and Faith-Based Organizations to Address the COVID-19 Pandemic in Collaboration with the United Nations. https://www.ohchr.org/EN/NewsEvents/Pages/DisplayNews.aspx?NewsID=25909\&LangID=E
} 
$(\mathrm{WHO})^{14}$ als auch Repräsentanten des Kinderhilfswerkes der UN (Unicef) ${ }^{15}$ auf die wichtige Rolle von religiösem Leitungspersonal und glaubensbasierten Organisationen bei der Bewältigung von weltweiten Krisen hin. Der Portugiese António Manuel de Oliveira Guterres, seit 2017 amtierender Generalsekretär der UN, betonte mehrfach, dass religiöse Akteure und religiöses Denken unbedingt bei nachhaltigen Entwicklungsfragen und in der Krisenprävention miteinzubeziehen seien. Dazu wurde 2017 sogar ein eigens Gremium, der Multi-Faith Advisory Council der UN, gegründet. In der aktuellen Covid-19- Situation hat sich diese in den internationalen Organisationen offensichtlich derzeit neu definierende Rolle von Religion in der Seuchenbewältigung und Krisenprävention fortgesetzt. ${ }^{16}$ Ob sich hiermit eine neue globale und internationale Wertschätzung von Religion durch die UN etabliert, die im Gegensatz zum sich vollziehenden Mitglieder- und Relevanzschwund der Kirchen in Westeuropa vollzieht, ist eine offene Frage.

Der Forschungsverbund versteht sich als ein offenes Netzwerk, das sich über Interesse, Kontakt, Forschungsthemen und weitere zukünftige Veranstaltungen und Aktivitäten freut. Kontaktadresse: Katharina Kunter, Professor Contemporary Church History, Faculty of Theology (University of Helsinki): Katharina.Kunter@helsinki.fi

\footnotetext{
${ }^{14}$ WHO, 2020: Practical considerations and recommendations for religious leaders and faith-based communities in the context of COVID-19, 7 April 2020, in: https://www.who.int/publications/i/item/practical-considerationsand-recommendations-for-religious-leaders-and-faith-based-communities-in-the-context-of-covid-19

${ }^{15}$ Welsh, Teresa, 2020: Faith leaders have key role in stopping spread of COVID-19, UNICEF says, Devex 30 July 2020. https://www.devex.com/news/faith-leaders-have-key-role-in-stopping-spread-of-covid-19-unicef-says$\underline{97821}$

${ }^{16}$ Vgl. hierzu u.a. Falk, Richard, 2001: Religion and Humane Global Governance. Basingstoke: Palgrave; James, Helen (Hg.), 2007: Civil Society, Religion and Global Governance. Paradigms of Power and Persuasion. New York: Routledge,Ager, Alastair, Ager, Joey, 2015: Faith, Secularism, and Humanitarian Engagement: Finding the Place of Religion in the Support of Displaced Communities. Basingstoke: Palgrave, Barnett, Michael, Stein, Janice, 2012: Sacred Aid: Faith and Humanitarianism. Oxford: Oxford University Press.
} 